\begin{tabular}{cc}
\hline & International Journal of Engineering \& Technology, $7(2.24)(2018) 536-540$ \\
SPC & International Journal of Engineering \& Technology \\
& Website: www.sciencepubco.com/index.php/IJET \\
Research paper & \\
\hline
\end{tabular}

\title{
Brain Tissue Classification using PCA with Hybrid Clustering Algorithms
}

\author{
Yepuganti Karuna ${ }^{1}$, Saritha Saladi ${ }^{2}$, Budhaditya Bhattacharyya ${ }^{3}$ \\ ${ }^{1,3}$ School of Electronics Engineering, VIT University, Tamil Nadu, INDIA \\ ${ }^{2}$ School of Electrical Engineering, VIT University, Tamil Nadu, INDIA \\ *Corresponding Author Email: karun@vit.ac.in
}

\begin{abstract}
Distinct algorithms were developed to segment the MRI images, to satisfy the accuracy in segmenting the regions of the brain. In this paper, we proposed a novel methodology for segmenting the MRI brain images using the clustering techniques. The Modified Fuzzy CMeans (MFCM) algorithm is pooled with the Artificial Bee Colony (ABC) algorithm after denoising images, features are extracted using Principal Component Analysis (PCA) for better results of segmentation. This improves the ability to extract the regions (cluster centres) and cells in the normal and abnormal brain MRI images. The comparative analysis of proposed methodology with existing FCM, ABC algorithms is evaluated in terms of Minkowski score. The proposed MFCM-ABC method is more robust and efficient to hostile noise in images when compared to existing FCM and ABC methods.
\end{abstract}

Keywords: ABC algorithm, FCM clustering, MRI, PCA, T1W-Brain images.

\section{Introduction}

Magnetic Resonance Imaging (MRI) produces a large volume of data, used by clinicians to diagnose the disease, pathology existing in the images [1]. MRI scan is a non-intrusive method, provides a good contrast between the tissues of the brain. During acquisition procedures images are affected by the undesirable signals such as noise non-illumination ailment and partial volume effect. The image segmentation is dividing an image into different anatomical structures based on the various parameters like regions, intensities, pixels, and volume. Different algorithms are developed to overcome the complexity incorporated in the process of segmentation and distinct algorithms are described in detail [2]. Various segmentation methods (thresholding, region growing, supervised, unsupervised and atlas-based methods).

Fuzzy C Mean (FCM) process is introduced by Dunn, 1973 and later revised by Bezdek, 1980. The implication of the fuzzy clustering allows the pixels based on their different intensity and membership values to form the clusters. It's an iterative process and sensitive to noise and other imaging artifacts. The most commonly used method is FCM, which is used for the sequential pattern mining process in data mining and provides the accurate performance when compared to $\mathrm{k}$ means clustering method [3].In segmentation of images, clustering is pondered to be image voxel as the data object and in that, each voxel is allocated to a cluster based on their similarity of selected features. The noise interfered is either gaussian or speckle noise and the image capturing equipment itself incorporates salt \& pepper noise. This noise degrades the images and leads to the incorrect diagnosis of the disease. Due to this coherent noise, it is more difficult to distinguish the adequate details of the images during the diagnosis of the disease by a human expert.

Denoising methods are intended to eliminate the noise from an image without losing the original attributes of the image.
Various denoising filters are bilateral, wavelet, principal component analysis, non-local means filters are used to exterminate noise from the MR images. The analysis of various denoising filter is carried out revealed that the SANLM filter provides the better results when compared to other existing filters [4]. Thus, denoising the noisy image has become the imperative step in processing the medical MRI images. Artificial Bee Colony is inspired by means of rummaging action of bees and treated as the meta-heuristic population-based algorithm [5]. Honey bees are social bugs and demonstrate features like bee rummaging, bee ballet, crowned head bee, chore collection, cooperative decision building, shell site choice, copulating, pheromone setting and steering systems, used as the replica of intellectual solicitations. It is simple, easy to employ, highly amenable, widely used by many researchers for various optimization problems [6]. This paper outlines methods and materials in section II, the proposed MFCM$\mathrm{ABC}$ is described in Section III, Section IV presents the results and discussion, Section $\mathrm{V}$ concludes the paper.

\section{Methods and Materials}

\section{A. Modified FCM (MFCM) Algorithm}

To overcome the limitations of conventional FCM algorithm, Modification in the membership function is defined as [9],

$$
\begin{aligned}
& x_{i j}^{\prime}=\frac{x_{i j}^{a} p_{i j}^{k}}{\sum_{t=1}^{k} x_{t j}^{a} p_{t j}^{k}} \\
& p_{i j}=\sum_{t \in N\left(u_{i}\right)} x_{i t}
\end{aligned}
$$

where $p_{i j}$-spatial function representing the probability of pixel $u_{i}$ belonging to $\mathrm{i}^{\text {th }}$ cluster, $\mathrm{N}\left(u_{i}\right)$-square window centered on pixel $u_{i}$ in spatial dominion and $a, k$ are parameters of window used. In a 
homogenous area, the spatial functions improve the innovative membership, and the clustering outcome residues unaffected. Nevertheless, for a noisy pixel, it will decrease the weighting of a noisy cluster by the labels of its neighboring pixel. As an outcome, misclassified pixels from noisy areas can be effortlessly amended. The objective function is:

$z_{a}=\sum_{i=1}^{k} \sum_{j=1}^{l} x_{i j}^{a}\left\|u_{i}-s_{j}\right\|$

$s_{j}=\frac{\sum_{i=1}^{k} x_{i j}^{a} u_{i}}{\sum_{i=1}^{k} x_{i j}^{a}}$

$\sum_{j=1}^{l} x_{i j}=1 ; \forall i, 0<\sum_{i=1}^{k} x_{i j}<k ; \forall k, x_{i j} \in[0,1] ; \forall i, j$

$$
x_{i j}=\frac{1}{\sum_{k=1}^{l}\left(\frac{\left\|u_{i}-s_{j}\right\|}{\left\|u_{i}-s_{k}\right\|}\right)^{\left(\frac{2}{a-1}\right)}}
$$

where $a$ - degree of fuzziness in clustering (in general $a=2$ ), $x_{i j}$ Membership of fuzzy data $u_{i}$ to $s_{j}$ as centre of the cluster and, $\left\|u_{i}-s_{j}\right\|^{2}$

- Distance between $u_{i}$ data and cluster centre $j, s_{j}$.

Algorithm steps of improved fuzzy C means:

Step 1: Set the number of clusters $n$ and the parameter $y$ in Eq (3). Initialize the fuzzy cluster centroid vector $U=\left\{u_{1}, u_{2}, \ldots . u_{i}, \ldots u_{l}\right\} ; u_{i} \in \mathfrak{R}_{d}$ randomly and set $\varepsilon=0.02$,

Step 2: compute $x^{\prime}{ }_{i j}$ using Eq (1),

Step 3: update $s_{j}$. using Eq (4),

Step 4: Repeat steps 2 and 3 until the resulting termination condition is satisfied:

$$
\max _{i j}\left\{x_{i j}^{q}-x_{i j}^{(q-1)}\right\}<\varepsilon
$$

Thus, the IFCM algorithm improves pointedly the performances by combing with other clustering methods.

\section{B. Artificial Bee Colony Algorithm}

Artificial Bee Colony is inspired by means of rummaging action of bees and treated as the meta-heuristic population-based algorithm [9]. Honey bees are social bugs and demonstrate features like bee rummaging, bee ballet, crowned head bee, chore collection, cooperative decision building, shell site choice, copulating, pheromone setting and steering systems, used as the replica of intellectual solicitations. It is simple, easy to employ, highly amenable, widely used by many researchers for various optimization problems [10].

$\mathrm{ABC}$ algorithm comprises of active bees, spectators, and spies Active bee is the bee going to find the victuals. Spectator bee waits on the dance region to acquire the info regarding victuals. The bee exploiting the random quest is termed as the spy bee. The location of a victuals indicates the feasible clarification to the optimization difficultly, and the nectar quantity of a victuals signifies the attribute of the concomitant solution. An erratically circulated population is generated initially. If the number of active bees and victuals is equal, then the locations are updated repetitively by subsequent cycles to reach the highest number of reiterations or to gratify the adjourn conditions. Every active bee recollects their former superlative location and yields a new-fangled location contained by its vicinity of their memory. If the obtained victuals are superior to previous victuals location then it updates by the new-fangled location. When the quest procedure is finished by all active bees and the report about the way, span of victuals and the nectar quantities are pooled thru spectators by a self-styled wiggle dance in their dancing region. In the surveillance of wiggle dance, every spectator selects a victuals reliant on the possibility and combines with the victuals, hunts the region in the interior to its neighbourhood to cause a new nominee solution. If a location cannot be enhanced after the determined number of cycles, the location is unrestrained and the corresponding active bees turn out to be a spy. The abandoned location is substituted with a newfangled erratically engendered victuals $[11,12]$.

\section{Proposed Methodology}

\section{A. Denoising}

The images are preprocessed using denoising filter and the block diagram of the proposed method is shown in figure 1 . The image used in implementing the proposed method is first denoised using Spatially Adaptive Non-Local Means (SANLM) filter improves the quality of the image. These filter deals with noise level spatially erratic in the image can be estimated locally by processing data with static or spatially varying noise fields in a fully automatic manner. This SANLM filters produce best results in removing noise from MR images when compared to other methods like bilateral, non-local means filters [7].Seizing the chromatic content of an image is termed as feature extraction. It is the process to epitomize the raw image in its reduced outline to simplify the decision making. In extracting the features from the image the most widely used application in medical image processing is Principal Component Analysis (PCA) [8]. In this paper, images are denoised using SANLM filter, extracted the features using PCA and then segmented the images using the Modified FCM correlated with $\mathrm{ABC}$ algorithms and these results are compared with the Minkowski score.

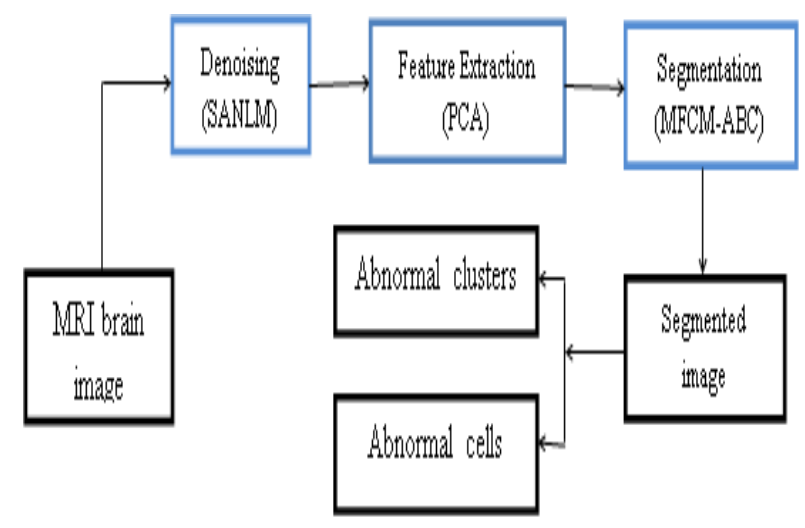

Fig. 1: Block diagram of the proposed method

\section{B. Modified FCM Clustering based ABC algorithm}

The automatic clustering method is technologically advanced to augment the process of brain segmentation using MRI, to rectify the limitations of Fuzzy $\mathrm{C}$ means algorithm. The Modified Fuzzy $\mathrm{C}$-means clustering is used with artificial bee colony algorithm to consequentially verify the accurate number of cluster centres, their locations, and abnormal cells in MRI images of multiple sclerosis lesions.

The pseudo-code of the proposed MFCM-ABC method:

1. $\quad$ Set the number of clusters $l$ and the parameter $u$ in Eq (3). Initialize the fuzzy cluster centroid vector randomly and set $\varepsilon$ $=0.02$.

2. Compute $x_{i j}^{\prime}$ using Eq (1), 
3. $\quad$ Update $s_{j}$. using Eq (4),

4. Repeat steps 2 and 3 until the resulting termination condition is satisfied: $\max _{i j}\left\{x_{i j}^{q}-x_{i j}^{(q-1)}\right\}<\varepsilon$, q is loop count. Thus, the MFCM algorithm improves pointedly the performances by combing with other clustering methods.

5. Initialize the population of $N p$ solutions, calculate the population at cycle $(\mathrm{G}=1)$

6. Repeat for new solution to determine the active bees

7. Employ gluttonous selection process and calculate the probability $P_{i}$

8. If the probability value $P_{i}$ is not in the matrix $Q_{i}$, then $Q_{i}=P_{i}$

9. Make new solutions for spectators, employ gluttonous selection process for spectators

10. Express the deserted solution and recollect the best victuals

11. Update the cycle with plus $1(\mathrm{G}=\mathrm{G}+1)$, these steps are repetitive till the maximum number of repetitions.

12. To determine the number of clusters $(\mathrm{R})$

for $\mathrm{i}=1: \mathrm{n}$; $\mathrm{n}$-width of all $\mathrm{n}$ images

for $j=1: m$; $m$-height of $n$ images

color $=\mathrm{c}$

if voxel $(\mathrm{i}, \mathrm{j}) . \mathrm{c}=$ pink color

disp ('color of the brain')

else if voxel $(i, j) . c \neq$ pink color

update $\mathrm{R}++$

end

end

end

end

The competence of the proposed MFCM-ABC algorithm is improved when compared with the existing FCMABC method [10] and it extracts the appropriate number of abnormal cells in every cluster automatically. This speeds up the computation probability in obtaining the intensities of clusters in the MRI images.

\section{Results and Discussion}

The performance of the proposed MFCM-ABC algorithm is validated after pre-processing with 100 iterations with colony size of 50 bees. The simulated 3D MRI brain images [11] are of volume $3 \mathrm{~mm}$ slice thickness, with $1 \times 1 \times 1 \mathrm{~mm}^{3}$ as voxel size and voxel resolution of $1 \mathrm{~mm}^{3}$, images of common size $181 \times 217 \times$ 181 voxels are used. MRI brain images are segmented to a number of clusters, the segmentation process of MFCM-ABC is attained by employing the intensity values of the voxel in the cluster as the feature space. The segmented images (output) are compared with the ground truth images. The obtained results are evaluated using quantization index with classification accuracy. The classification accuracy is calculated by the similarity index of the clustered image and the ground truth image.

The Minkowski Score (MS) is used as quantization index to evaluate the performance of the proposed method by making use of classification accuracy rate [12] . The calculation of MS uses the following equation

$M S(x, y)=\sqrt{\frac{s_{01}+s_{10}}{s_{11}+s_{10}}}$

where $\mathrm{x}$ and $\mathrm{y}$ are the partition matrices of ground truth image and the segmented image. $S_{01}$ and $S_{10}$ indicates the number of element pairs belongs to $\mathrm{y}$ and $\mathrm{x}$ in the same cluster respectively. $S_{11}$ indicates the pair of elements in both $\mathrm{x}$ and $\mathrm{y}$ belonging to the same cluster. The optimum value of the Minkowski Score is zero and lower values signify the compact and well-separated cluster which proves that segmented results are more accurate. In the proposed MFCM-ABC method the best matched least value in between the segmented and ground truth images are to be considered.

The simulated 3D data consist of normal and abnormal subjects with different slice thickness, noise, and intensity non-uniformity. Experiment has performed on various subjects with different noise levels $(0,1,3,5,7,9) \%$ and intensity non-uniformity $(0,20,40) \%$ and the proposed algorithm effectively determines the number of abnormal cluster and cells in normal MRI images are tabulated in Table 1. The evaluation of the Minkowski score of the proposed MFCM-ABC method and FCMABC [10] are differentiated in fig 2 (a).

Table 1: Average values MS for T1-weighted MR images of the normal brain images

\begin{tabular}{|c|c|c|c|}
\hline $\begin{array}{c}\text { NORMAL } \\
\text { IMAGES }\end{array}$ & $\begin{array}{c}\text { MS OF } \\
\text { FCM }\end{array}$ & $\begin{array}{c}\text { MS OF } \\
\text { FCMABC }\end{array}$ & $\begin{array}{c}\text { MS OF } \\
\text { PROPOSED }\end{array}$ \\
\hline Slice-40 & 1.2453 & 0.7424 & 0.6052 \\
\hline Slice-51 & 1.3298 & 0.6829 & 0.5341 \\
\hline Slice-64 & 1.3954 & 0.5887 & 0.5030 \\
\hline Slice-91 & 1.1325 & 0.6186 & 0.5278 \\
\hline Slice-102 & 0.9523 & 0.6186 & 0.5278 \\
\hline
\end{tabular}

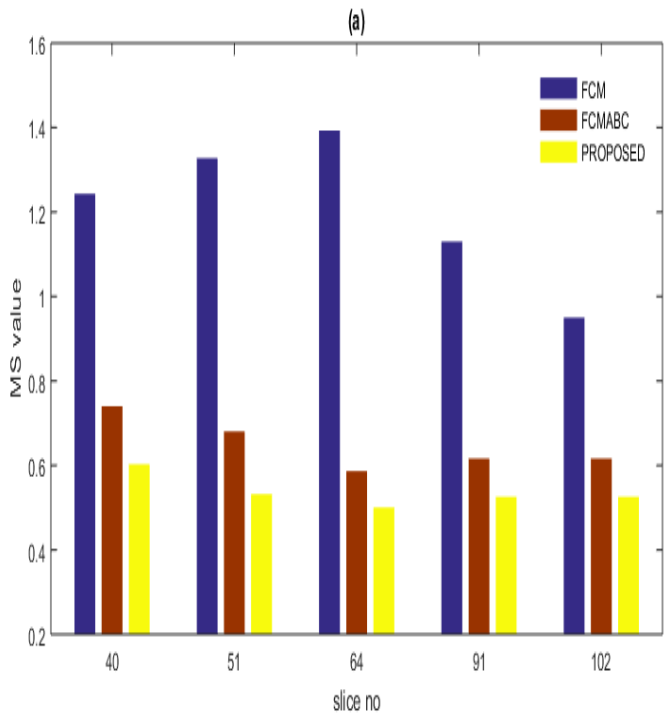

(b)

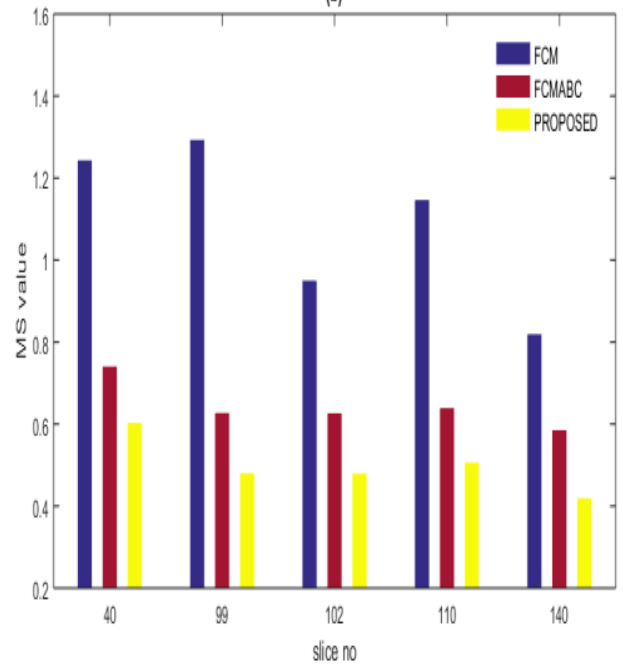

Fig. 2: Minkowski Score comparison (a) Normal and (b) Abnormal MRI images 
The evaluation of the Minkowski score of the proposed MFCMABC method and FCMABC [10] are differentiated in fig 2 (b) and with the tabulation of abnormal cells and clusters separately in table 2. The Minkowski score for the normal MRI brain image of slice 102 is improved $30.6 \%$ by the proposed MFCM-ABC method, proves that proposed method is efficient in tissue classification when compared to FCMABC method [10].
The simulated 3D MRI brain images from brain web 2003 are used in generating the comparative study between the proposed and the existing method. In fig 4 the abnormal brain image of slices 110 and 99 are produced with quantitative analysis of the Minkowski score is compared among the ground truth and the segmented image by proposed MFCM-ABC algorithm after preprocessing and extracting the features.

Table 2: Average values MS for T1-weighted MR images of the abnormal brain images

\begin{tabular}{|c|c|c|c|}
\hline $\begin{array}{c}\text { ABNORMAL } \\
\text { IMAGES }\end{array}$ & $\begin{array}{c}\text { MS OF } \\
\text { FCM }\end{array}$ & $\begin{array}{c}\text { MS OF } \\
\text { FCMABC }\end{array}$ & $\begin{array}{c}\text { MS OF } \\
\text { PROPOSED }\end{array}$ \\
\hline Slice-40 & 1.2453 & 0.7424 & 0.6052 \\
\hline Slice-99 & 1.2954 & 0.6291 & 0.4823 \\
\hline Slice-102 & 0.9523 & 0.6285 & 0.4812 \\
\hline Slice-110 & 1.1486 & 0.6412 & 0.5078 \\
\hline Slice-140 & 0.8214 & 0.5874 & 0.4212 \\
\hline
\end{tabular}

Slice no 99

Slice no 102

Slice no 103
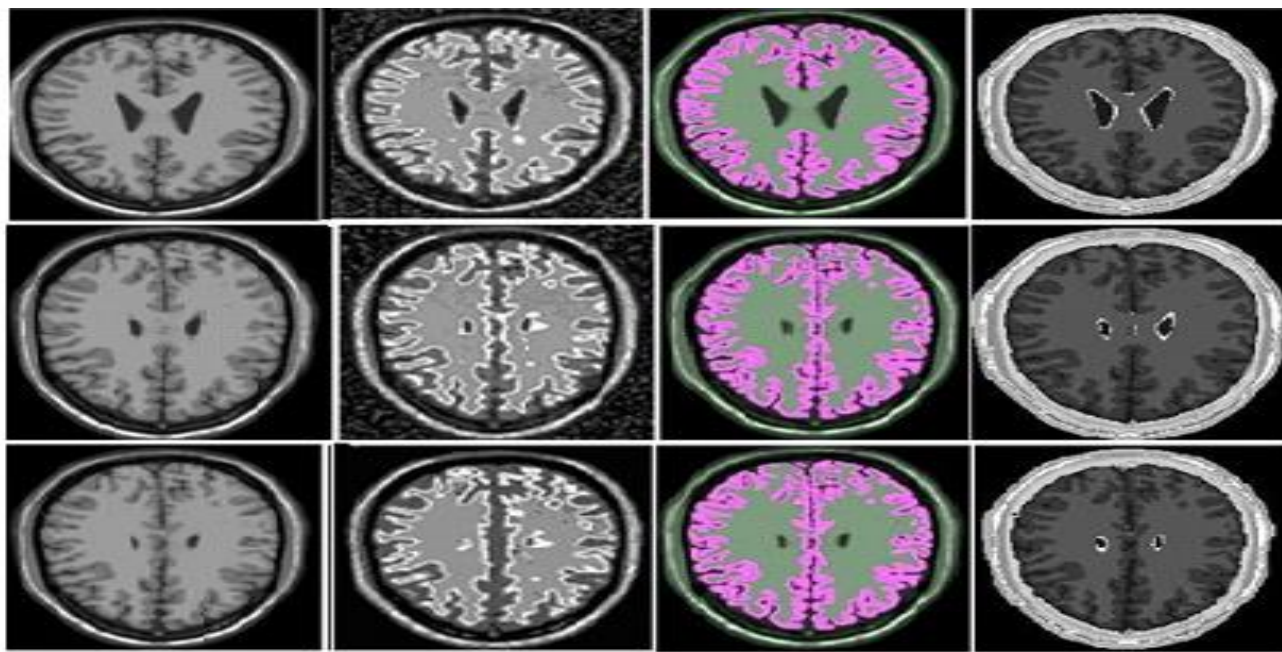

Slice no 110
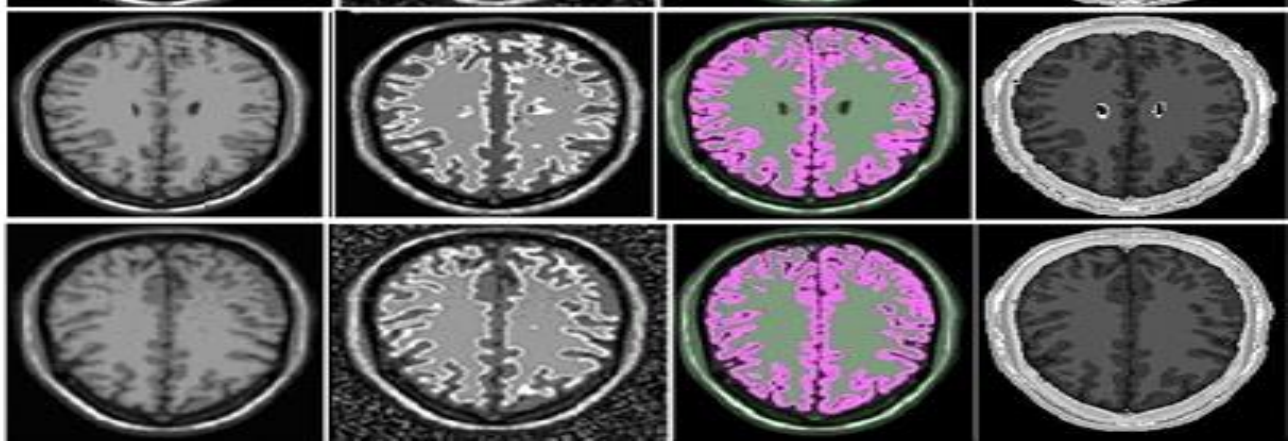

Fig. 3: Simulated MRI brain images (a) Abnormal brain image data(original) (b) Segmented FCMABC output (c) Segment output by proposed method (d) Ground truth image

The Minkowski Score for the normal MRI brain image of slice 110 is improved by $90 \%$ by the proposed MFCM-ABC method, proves that proposed method is more robust when compared to the conventional FCM method. The results acquired by the proposed method are pointing towards the success in ascertaining the abnormal cells and clusters when compared with the ground truth images. The intended MFCM-ABC method is effective in segmenting the MRI brain images and voxels of the cells and clusters are determined more efficiently with the help of PCA feature extraction method.

\section{Conclusion}

The efficient and trustworthy clustering based segmentation method is proposed. The foremost step is pre-processing of MRI brain images and from those denoised features are extracted using PCA. Likewise, the capability of the proposed method helps in avoiding the vulnerability of the traditional FCM method where convergence rate and local minima get stuck. Traditional FCM clustering algorithm accomplishes very fast and diffident, but it does not assure high accuracy and computational complexity is high for noisy and the abnormal brain MRI images. To increase the accuracy $\mathrm{ABC}$ algorithm is employed in the proposed methodology to determine quicker optimal finding solution in the segmentation of the brain images. The proposed method is implemented and the results show that with lower the Minkowski score are obtained with and improvement by a mean of $20 \%$ for the proposed method when compared to existing algorithms. Thus, the experimentation shows the efficiency of the proposed MFCM$\mathrm{ABC}$ algorithm on simulated MRI brain images is high in determining the appropriate number of abnormal cells and clusters.

\section{References}

[1] Jones, Rachel. "Neurogenetics: What makes a human brain?"Nature Reviews Neuroscience 13.10 (2012): 655-655

[2] Saritha, Saladi, and N. AmuthaPrabha. "A comprehensive review: Segmentation of MRI images-brain tumor." International Journal of Imaging Systems and Technology 26, no. 4 (2016): 295-304

[3] Selvanambi, Ramani, and Jaisankar Natarajan. "Cyclic Repeated Patterns in Sequential Pattern Mining Based on the Fuzzy CMeans Clustering and Association Rule Mining Technique." 
[4] Saladi, Saritha, and N. Amutha Prabha."Analysis of denoising filters on MRI brain images", International Journal of Imaging Systems and Technology 27, no. 3 (2017): 201-208.

[5] Karaboga, Dervis, and Celal Ozturk. "A novel clustering approach: Artificial Bee Colony (ABC) algorithm." Applied soft computing 11.1 (2011): 652-657.

[6] Abraham, Ajith, Ravi Kumar Jatoth, and A. Rajasekhar. "Hybrid differential artificial bee colony algorithm", Journal of computational and theoretical Nanoscience 9.2 (2012): 249-257.

[7] Manjón, José V., et al. "Adaptive non-local means denoising of MR images with spatially varying noise levels." Journal of Magnetic Resonance Imaging31.1 (2010): 192-203.

[8] Takao, Hidemasa, Naoto Hayashi, and Kuni Ohtomo. "Brain morphology is individual-specific information." Magnetic resonance imaging 33, no. 6 (2015): 816-821.

[9] Wang, Ping, and HongLei Wang. "A modified FCM algorithm for MRI brain image segmentation." In Future BioMedical Information Engineering, 2008. FBIE'08. International Seminar on, pp. 26-29. IEEE, 2008.

[10] Alsmadi, Mutasem K. "Mri brain segmentation using a hybrid artificial bee colony algorithm with fuzzy-c mean algorithm." Journal of Applied Sciences15.1 (2015): 100.

[11] BrainWeb, 2003. BrainWeb: Simulated MRI volumes for normal brain.Mcconnell Brain imaging centre, Montreal Neurological institute, Mcgill University.

[12] Xu, Shuo, Xiaodong Qiao, Lijun Zhu, Yunliang Zhang, Chunxiang Xue, and Lin Li. "Reviews on Determining the Number of Clusters." Applied Mathematics and Information Sciences 10, no. 4 (2016): 1493-1512.

[13] T. Padmapriya and V. Saminadan, "Priority based fair resource allocation and Admission Control Technique for Multi-user Multi-class downlink Traffic in LTE-Advanced Networks", International Journal of Advanced Research, vol.5, no.1, pp.1633-1641, January 2017.

[14] S.V.Manikanthan and T.Padmapriya "Recent Trends In M2m Communications In 4g Networks And Evolution Towards 5g", International Journal of Pure and Applied Mathematics, ISSN NO:1314-3395, Vol-115, Issue -8, Sep 2017.

[15] S.V. Manikanthan , T. Padmapriya "An enhanced distributed evolved node-b architecture in 5G tele-communications network" International Journal of Engineering \& Technology (UAE), Vol 7 Issues No (2.8) (2018) 248-254.March2018. 